\title{
Laparoscopic Myomectomy for the Removal of Large Uterine Myomas
}

\author{
Rikiya Sano, MD, $\mathrm{PhD}^{1}$ Soichiro Suzuki ${ }^{1}$ Mitsuru Shiota, MD, $\mathrm{PhD}^{1}$ \\ 1 Department of Gynecologic Oncology, Kawasaki Medical School, \\ Kurashiki, Okayama, Japan \\ Surg J 2020;6(suppl S1):S44-S49. \\ Address for correspondence Rikiya Sano, MD, PhD, Department of \\ Gynecologic Oncology, Kawasaki Medical School, 577 Matsushima, \\ Kurashiki, Okayama, Japan (e-mail: sano_r@med.kawasaki-m.ac.jp).
}

\author{
Abstract \\ Keywords \\ - laparoscopic \\ myomectomy \\ - myomas \\ - laparoscopic surgery \\ - malignant tumor \\ - surgical procedure \\ - fibroids
}

\section{Preoperative Evaluation and Imaging}

As with preoperative examination of normal myomas, magnetic resonance imaging (MRI) is recommended in addition to internal examination and ultrasonography for giant myomas. In particular, MRI is important in precluding uterine malignancy and adenomyosis. It is also highly effective in determining the size of the myoma, site of development, shape, number, presence or absence of degeneration, and height of the uterus fundus. It is also highly important in estimating the distance to the serosa, and the distance from the nodule to the endometrium, which are both important factors for performing a safe and effective procedure.

\section{Indication/Contraindication}

First, it is necessary to exclude any uterine malignant tumors from the clinical course and diagnostic imaging findings. Moreover, in the case of giant myomas, attention must be paid to any difficulty that may occur in securing the visual field. Furthermore, clinicians should be aware of and plan for any unexpected bleeding as the diameter of the myomas increases. Moreover, in many cases of laparoscopic myomec- tomy (LM), the intraoperative bleeding volume defines the adaptation limit of surgery. In this respect, Dubuisson et al ${ }^{1}$ reported that the nucleus size is $\leq 8 \mathrm{~cm}$ and the number of nodules is $\leq 2$, and Takeuchi et $\mathrm{al}^{2}$ reported that the amount of intraoperative bleeding significantly increases when the maximum myoma size exceeds $10 \mathrm{~cm}$. Furthermore, the American College of Obstetricians and Gynecologists in 2008 recommended that in the case of a uterine myoma of 5 to $8 \mathrm{~cm}$ in size with multiple uterine nodules and deep interstitial myoma, it is necessary to perform laparotomy or laparoscopic-assisted surgery only in accordance with the operator's skill. Our previous study reported that intraoperative bleeding significantly increased in cases in which the maximum size of the nodule exceeded $10 \mathrm{~cm}$. Hence, we suggest that there may be no restriction on the number of the nodules, but in cases in which the myoma size exceeds $10 \mathrm{~cm}$, a combination of small incisions should be considered to mitigate some of the associated surgical risks.

In addition, gonadotropin-releasing hormone (GnRH) agonist therapy prior to LM has been recommended, as it has been shown to reduce myoma size, amount of intraoperative bleeding, and operation time. However, while long-term administration of this therapy may result in the degeneration of the
DOI https://doi.org/ 10.1055/s-0039-1694989. ISSN 2378-5128.
Copyright $\odot 2020$ by Thieme Medical Publishers, Inc., 333 Seventh Avenue, New York, NY 10001, USA. Tel: +1(212) 760-0888.
License terms

()(1) $\circledast$ 
myoma, it can also make complete myomectomy difficult due to the presence of small myomas after administration of the therapy. This could then decrease the efficacy of the procedures; hence, more research needs to be conducted to prove or disprove the efficacy of GnRH agonist therapy in these cases.

\section{Preoperative Preparation}

Although almost all cases do not require blood transfusion in LM, in preparation for possible bleeding, clinicians should consider preparing autologous blood and Cell Saver on a case-by-case basis.

\section{In-Depth Explanation}

\section{Pneumoperitoneum and Installation of a Primary Trocar}

The authors perform all laparoscopic surgery with pneumoperitoneum (-Fig. 1). Normally, insufflation is performed from the umbilicus portion using a closed entry technique, and a 5-mm primary trocar is used to create the location where the camera port is inserted. In cases in which the uterus itself is large, it is necessary to pay special attention to not piercing it. In cases of giant myoma where the uterus base exceeds the umbilical height, it is also necessary to first insert a trocar above the umbilicus or insert a trocar at Palmer's point under the optical view following pneumoperitoneum.

When performing laparoscopically assisted myomectomy (LAM), first, a small incision of approximately 3 to $5 \mathrm{~cm}$ is made in the lower abdominal wall; then, insuflation becomes possible by installing trocar mounting cuffs such as a Lap Disc. While observing the inside of the abdominal cavity, a trocar is inserted for the camera port at an appropriate position (navel position or above) according to the size of the uterus.

\section{Tips and Warnings}

In cases in which the myomas are extremely large, pulling of the nucleus toward the patient's head side is indispensable. Furthermore, the first trocar may be installed at least $3 \mathrm{~cm}$ above the height of the uterus fundus to secure the field of vision. In addition, the authors establish pneumoperitoneum at Palmer's point $(3 \mathrm{~cm}$ below the ribs, on the middle of the left clavicle) in cases of giant myomas or in patients with a history of abdominal surgery and expected intraperitoneal adhesions ( - Fig. 1).

\section{Intraperitoneal Observation}

Insert the laparoscope and observe the entire abdominal cavity. Confirm the position and size of the myoma and the presence of adhesions between the uterus and adjacent organs, and assess whether an LM procedure is possible. If it is judged that it may be difficult to accomplish LM, consider making a small incision and converting to LAM. Even in cases in which LAM is judged to be too difficult and a laparotomy is considered, determining the state of the abdominal cavity and the myoma by a laparoscope helps to predict the minimum abdominal wall incision length necessary for performing the operation. Furthermore, when adhesions between the uterus and adjacent organs are present, it is also possible

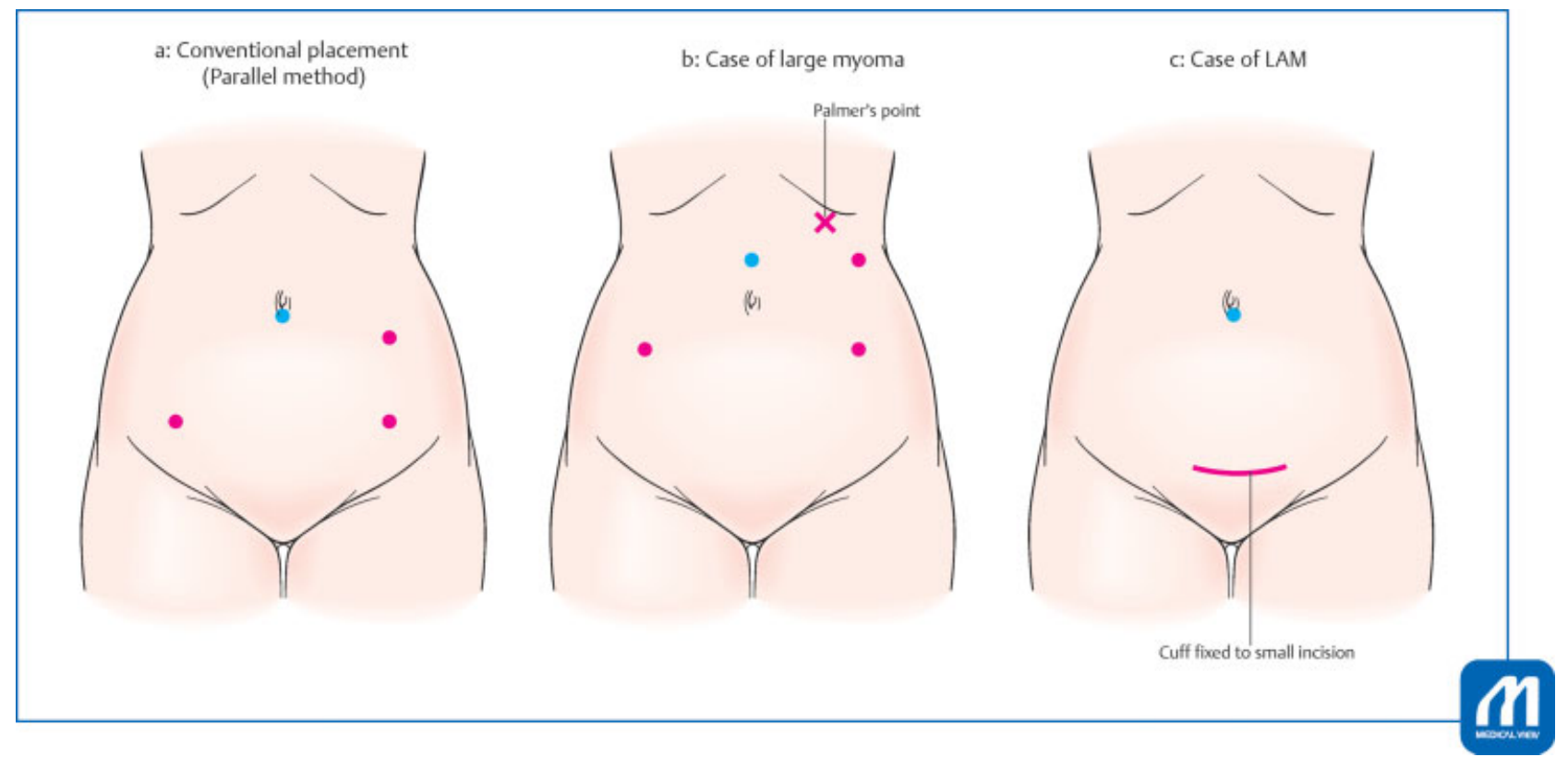

Fig. 1 (a) is conventional parallel method. (b) is trocar placement in case of large myoma. If the first trocar at umbilicus may puncture the uterus, first of all, you try to insufflate at Palmer's point and optically put trocars in the right position. (c) is case of LAM. (Reproduced with permission from Umemoto M, Kotani Y. Shiota M. In: Hiramatsu Y, Konishi I, Sakuragi N, Takeda S, eds. Mastering the Essential Surgical Procedures OGS Now, No.11. Uterine Myoma: How to Operate in These Cases? (Japanese). Tokyo: Medical View; 2012: 18-25. Copyright @ Medical View.) 
to perform adhesion detachment under laparoscopy in advance.

\section{Tips and Warnings}

In the case of giant myomas, the uterus is often rotated. In laparoscopic surgery, there is a restriction on the direction in which the clinician's hand can move when suturing the uterine wound site. Therefore, it is necessary to check the rotation of the uterus in advance since the direction of the uterine wound site after dissection of the nodule could be an important limitation. It is possible to check the degree of rotation by identifying the attachment positions of the uterine portion of the round ligament, fallopian tubes, and unique ovarian ligaments on both sides.

\section{Trocar Placement}

Our standard port placement method is the parallel method. A 5-mm forceps port is installed in the vicinity of the McBurney point, $12 \mathrm{~mm}$ on the side of the McBurney point, and $5 \mathrm{~mm}$ on the opposite side on the head side (i.e., upper left abdomen at roughly the height of the umbilicus), and the surgeon performs a standing operation on the left side of the patient. Depending on the facility, $12-\mathrm{mm}$ trocars may be installed in the left upper abdomen, and the surgeon may be standing on the right side of the patient and then the trocars must be reversed. In a facility where the surgery is performed by the diamond method, instead of the port being placed on the upper left abdomen, there have been cases in which the port is installed at the lower abdominal midline.

\section{Tips and Warnings}

When the uterus is large, and a camera port is placed on the head side rather than the umbilicus to secure the visual field, the forceps port may also need to be installed on the head side to maintain the triangulation of forceps and target organs (-Fig. $\mathbf{1}$ ).

\section{Injection of Vasopressin}

Injection of vasopressin facilitates a reduction in bleeding, which can occur because of the contractile action of the uterine smooth muscle as well as the enucleating operation of the myoma. It is common to directly inject vasopressin into the myometrium surrounding the myoma. Approximately, 5 to 20 units of vasopressin ( 20 units $=1$ ampule mixed in $100-\mathrm{mL}$ saline) have been used previously per nodule. Once the injection is administered, attention must be paid to any elevations in blood pressure, bradycardia, or coronary artery spasm caused by the pharmacological action of vasopressin.

\section{Incision of the Myometrium}

Incise the uterus surface at the myoma site with an energy device. The incision direction may be either transverse or longitudinal, but it is generally accepted that transection using a transverse incision causes less damage, considering the orientation of the vasculature (arcuate artery and radial artery) in the uterine body. Moreover, depending on the installation position of the trocar, the hand movement direction at the time of uterine suturing will be different. Therefore, it is important to select the direction of incision while considering the wound suture operation. Since the authors are performing surgery by parallel method, in principle, the incision of the uterus should be transected transversely and sutured vertically. By either method, a sufficiently large incision should be made to smoothly remove the nodules. If the incision size is not appropriate, it takes additional time to dissect and remove the nodule and then the transition to the suture hemostasis operation is delayed, which can increase the amount of bleeding. Furthermore, confirmation of the bottom portion of the myometrium becomes more difficult, and it is easy to make dead space. Bleeding from the active bleeding point of the wound is terminated using bipolar coagulation; however, it is most important to incise the myometrium in a short time to suppress bleeding.

\section{Tips and Warnings}

To reduce bleeding, it is important to peel off the capsular layer as close as possible to the nodule. To distinguish this layer, it is necessary to incise deeply so that the incision is sufficiently inside the nodule (-Fig. 2 ). If the incision is shallow, and the exfoliation layer between the nodule and the normal myometrium is not appropriate, it may cause unnecessary bleeding after myomectomy.

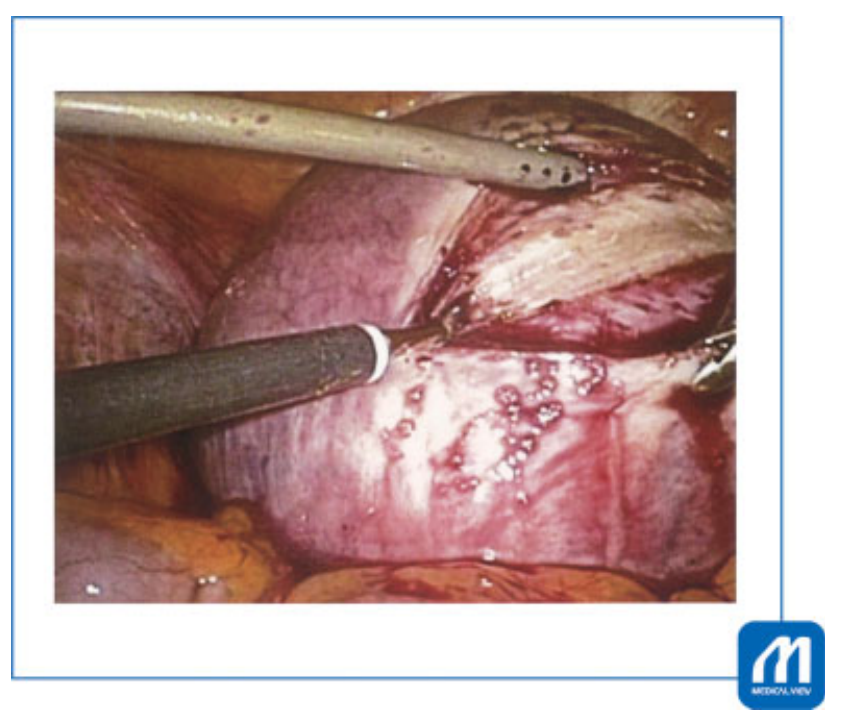

Fig. 2 The incision should be a sufficiently large to remove the nodule smoothly. And It is better to make an incision to cut into nodule to recognize right muscle layer. (Reproduced with permission from Shiota M, Umemoto M, Sano R. In: Hiramatsu Y, Konishi I, Sakuragi N, Takeda S, eds. Mastering the Essential Surgical Procedures OGS Now, No.19. (Japanese). Tokyo: Medical View; 2014: 142-151. Copyright @ Medical View.) 


\section{Myomectomy}

By using corkscrew manipulation and pulling the nodule, it is possible to suppress the bleeding by compressing the incised

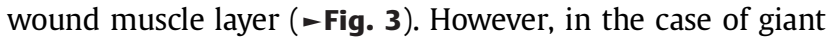
myomas, since the working space is narrow and there are restrictions on the pulling direction of the nodule, dissection progression should occur so as to roll the nodule while appropriately changing the hooking position of the corkscrew. The energy device should be used properly, and the device should advance across the normal myometrium. At this time, if the corkscrew is not hooked at an appropriate depth, it is important to be aware that adjacent organs may be damaged due to unexpected sliding. Conversely, if the hooking is too deep, care must be taken to not penetrate the myoma and expose the tip. This is especially true in cases of degenerative myomas, where caution is necessary for smoothing of the corkscrew.

In the case of LAM, it is also possible to conduct enucleation manually from a small incision. Since peeling while also pulling the nodule in LM or laparotomy is difficult, grasping and pulling the uterine muscle layer surrounding the incised myoma can assist in the process in this case. Then, pulling it with the hand also on the operation site should help guide it just under the small incision. In many cases of giant myoma, the nodule is in contact with the endometrium, and efforts should be undertaken to avoid damaging this tissue as much as possible. In the case of endometrial damage, perform continuous suturing repair of the endometrium using a 3-0 absorbable thread. Since hemostasis can be difficult to achieve if a hematoma is formed between the endometrium and the muscular layer after myomectomy, the authors have also performed shallow suturing of the endometrium in the first layer of myometrium suturing.

\section{Tips and Warning}

Myoma degeneration can be significant, and often towing of the nodule is not sufficient. Even with strongly degenerative myomas, the myomatous capsule is often somewhat stronger. It is, therefore, advisable to enlarge the incision, preserve the capsule as much as possible, and peel the normal muscle layer back along the nodule with less traction force.

\section{Wound Suturing}

Immediately after myomectomy, the uterine wound site is sutured hemostatically with a 1-0 absorbable thread. In the case of giant myomas, the bleeding rate from the wound face may be high, but it is often controlled by Z-suturing at the bottom of the wound ( - Fig. 4). Moreover, care should be taken to not form a postoperative hematoma in the dead space, as the wound depression will become larger as a result. To suture so as not to create dead space, it is necessary to suture the muscle layer in two to four layers by Z-suturing, single ligation suturing, or continuous suturing according to the individual case, while also checking the bottom of the wound. In addition, if there is an excessive uterine capsule and it is difficult to close the wound site, trimming may be performed, as necessary.

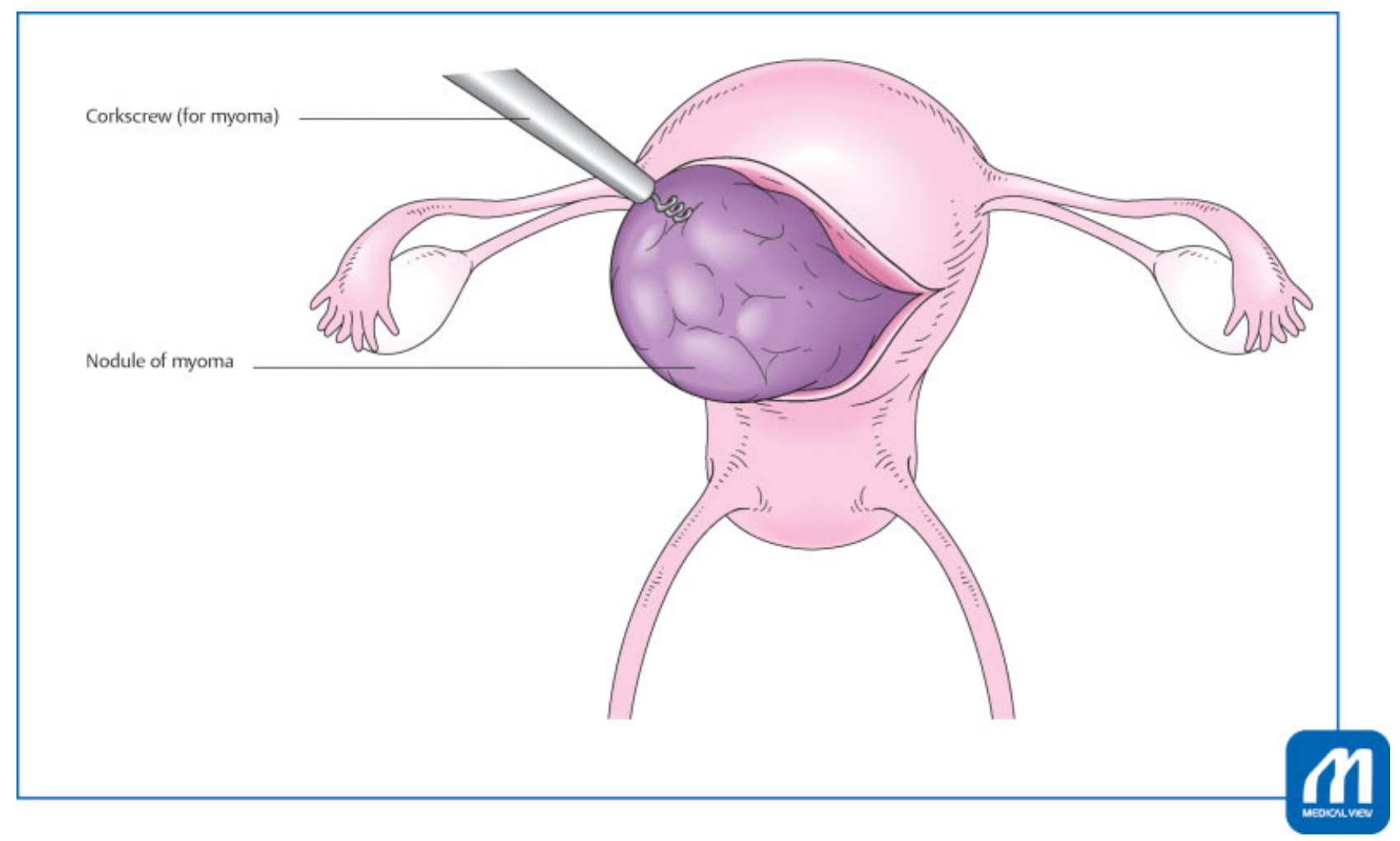

Fig. 3 Traction the uterus using corkscrew, the boundary between myoma and normal muscle layer can be easily recognized and nucleation can be performed smoothly. (Reproduced with permission from Umemoto M, Kotani Y. Shiota M. In: Hiramatsu Y, Konishi I, Sakuragi N, Takeda S, eds. Mastering the Essential Surgical Procedures OGS Now, No.11. Uterine Myoma: How to Operate in These Cases? (Japanese). Tokyo: Medical View; 2012: 18-25. Copyright @ Medical View.) 


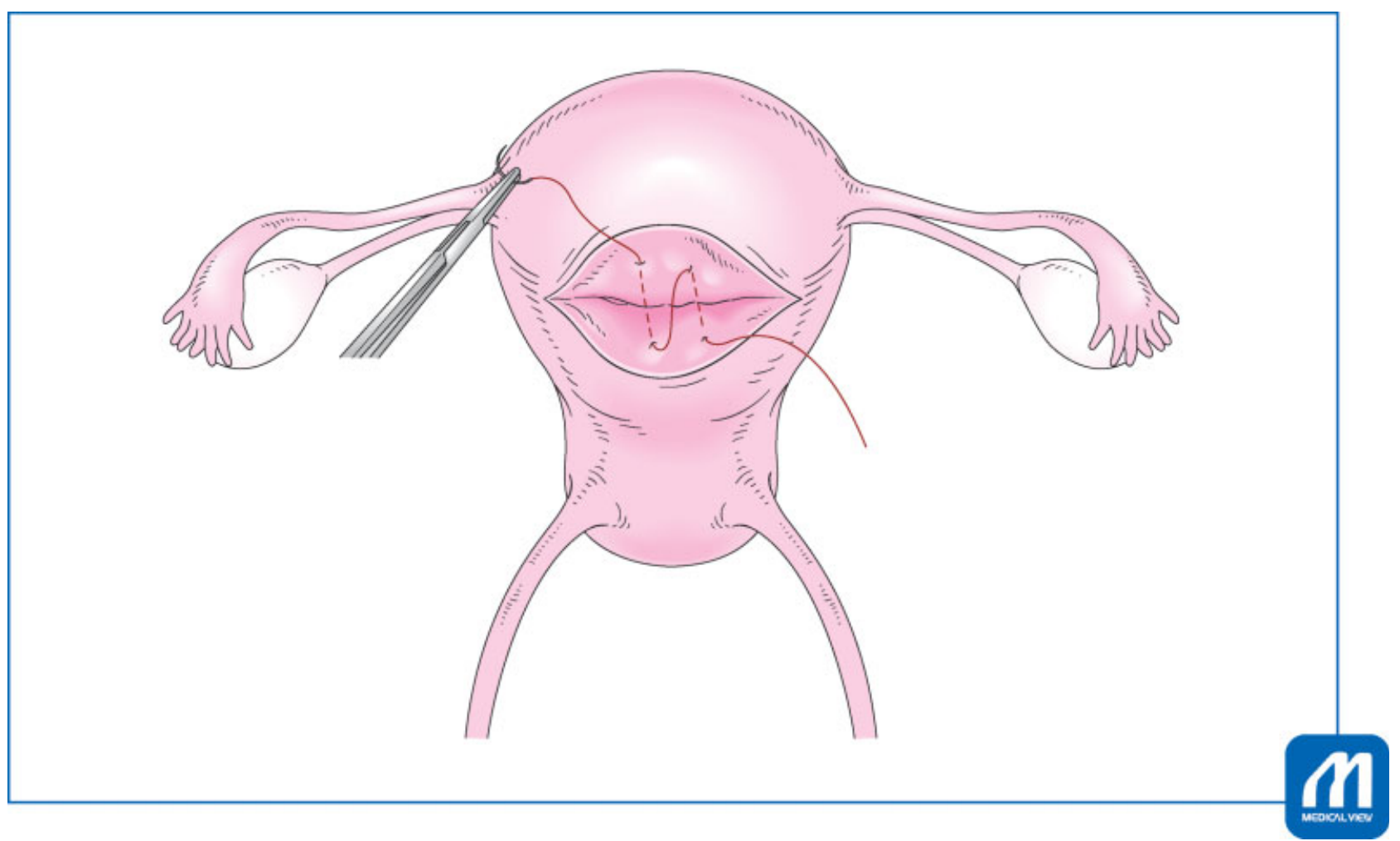

Fig. 4 Z suturing of the bottom. In many cases, this suturing can reduce bleeding and prevent dead space formation. (Reproduced with permission from Umemoto M, Kotani Y. Shiota M. In: Hiramatsu Y, Konishi I, Sakuragi N, Takeda S, eds. Mastering the Essential Surgical Procedures OGS Now, No.11. Uterine Myoma: How to Operate in These Cases? (Japanese). Tokyo: Medical View; 2012: 18-25. Copyright @ Medical View.)

\section{Tips and Warnings}

In cases of giant subserosal myomas where the stem is visible, coagulation and cutting after ligating the stem will reduce the amount of bleeding. However, if a thick nutrient blood vessel is present, the operation may result in unexpected bleeding (-Fig. 5). In addition, the ligature in the stem is often loose, and it is necessary to ligate again after dissecting the myomas. This prevents further postoperative bleeding due to suture thread slipping.

\section{Extraction of the Myoma}

The myoma is extracted using a scalpel or power morcellator while cutting (-Fig. 6). Depending on the available facilities, the cut myoma may be removed using the opened Douglas fossa. In the case of LAM, it is possible to extract a myoma using a scalpel or a cooper under direct vision from a small incision.

It is desirable to extract the nodules in the same way in which you might peel an apple. When using a power morcellator in cases of giant myomas, the tip may not be visually confirmed in some cases. If this occurs, tow the claw forceps grasping the tissue while also fixing the camera and morcellator. The insertion depth of the power morcellator should absolutely not be changed.

In case of giant myomas, the surgeon may have to perform morcellation under conditions in which sufficient working space cannot be secured. In these cases, special attention should be paid specifically to not damage the intestines. Care must also be taken to prevent the generation of parasitic myomas due to the remnants of fibrous debris when using powered morcellation.

\section{Tips and Warnings}

To date, the use of a powered morcellator is widespread. However, in April 2014, a notice was released on the use of powered morcellators for laparoscopic hysterectomy or myomectomy by the U.S. Food and Drug Administration (FDA). The report specifically focused on cases of laparoscopic hysterectomy or myomectomy for a myoma diagnosed as sarcoma postoperatively. The report suggested that operations conducted using a power morcellator had the unexpected risk of dissemination of cancerous tissues. At this point, the FDA no longer recommends laparoscopic hysterectomy or myomectomy using a power morcellator. When considering whether to use a morcellator, a concerted effort should be made to ensure exclusion of malignant diagnosis using imaging tools such as MRI prior to surgery. In our facility, we adopted an in-bag power morcellation technique in an effort to prevent any tissue scattering. There are several disadvantages of this technique, including the time it takes to install the bag and assisting forceps cannot be used in the bag. However, overall it is a very useful method with respect to safety concerns, especially with practice and when performed correctly ( - Fig. 7). 


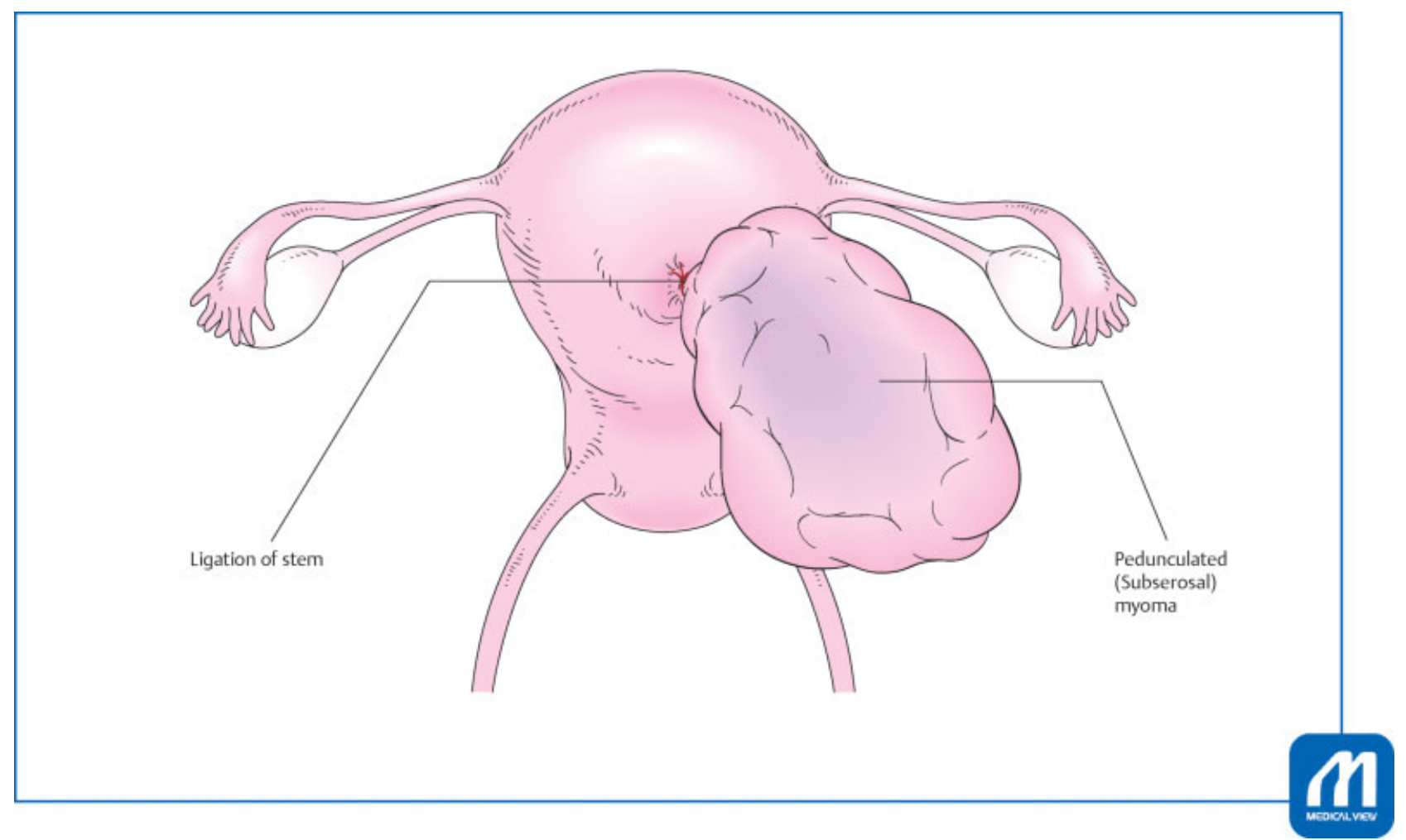

Fig. 5 By cutting the stem after ligation, the amount of bleeding can be reduced. If the stem is thick, it is safe to add ligation again after cutting, since the suture thread may be loosened or slipped after cutting. (Reproduced with permission from Umemoto M, Kotani Y. Shiota M. In: Hiramatsu Y, Konishi I, Sakuragi N, Takeda S, eds. Mastering the Essential Surgical Procedures OGS Now, No.11. Uterine Myoma: How to Operate in These Cases? (Japanese). Tokyo: Medical View; 2012: 18-25. Copyright @ Medical View.)

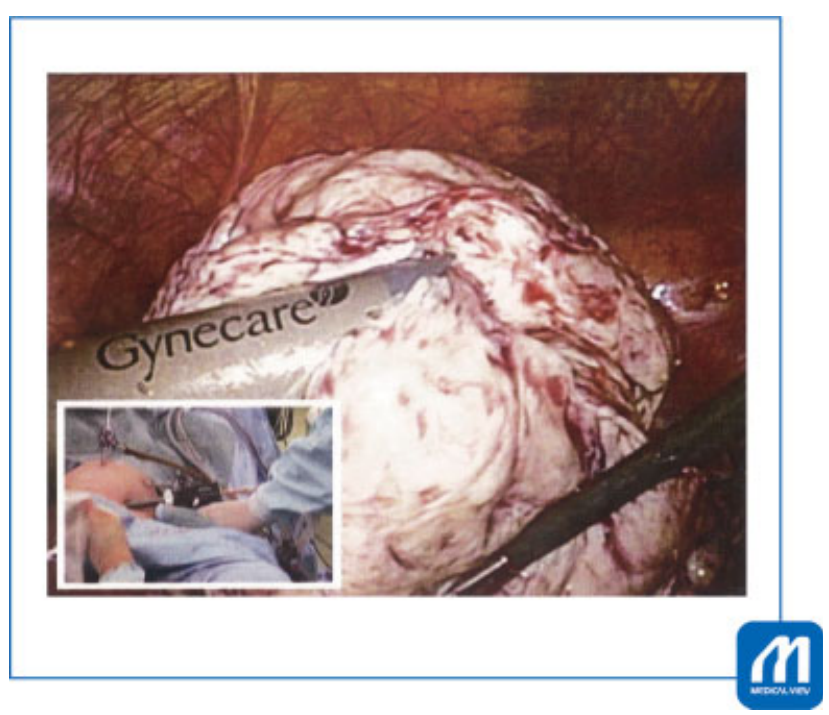

Fig. 6 The most important thing to prevent damage to surrounding organs is to fix the camera and morcellator. (Reproduced with permission from Shiota M, Umemoto M, Sano R. In: Hiramatsu Y, Konishi I, Sakuragi N, Takeda S, eds. Mastering the Essential Surgical Procedures OGS Now, No.19. (Japanese). Tokyo: Medical View; 2014: 142-151. Copyright (c) Medical View.).

\section{Hemostasis Check}

Confirm hemostasis at the uterine wound and perform bipolar coagulation and additional suturing, as necessary. To wash the abdominal cavity with physiological saline and prevent adhesion to the uterine wound site, adhesion prevention agents

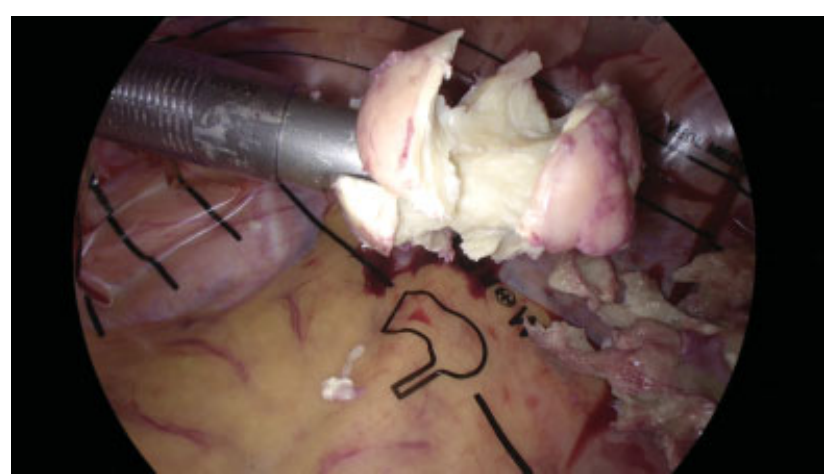

Fig. 7 In bag power morcellation can prevent tissue scattering.

(Seprafilm, Interceed, AdSpray) should be used, especially for patients who desire subsequent childbirth.

\section{Conflict of Interest \\ None.}

\section{References}

1 Dubuisson JB, Fauconnier A, Babaki-Fard K, et al. Laparoscopic myomectomy: a current view. Hum Reprod Update 2000; 6:588-594

2 Takeuchi H, Kuwatsuru R. The indications, surgical techniques and limitations of laparoscopic myomectomy. JSLS 2003;7:89-95 